\title{
Role of stress in modulation of skin neurogenic inflammation
}

\author{
OVIDIU GRIGORE ${ }^{1}$, ALEXANDRA IOANA MIHAILESCU $^{2}$, IULIA SOLOMON $^{3}$, \\ DANIEL BODA $^{4,5}$ and CONSTANTIN CARUNTU ${ }^{5,6}$
}

\author{
${ }^{1}$ Department of Applied Electronics and Information Engineering, Polytechnic University of Bucharest, 061071 Bucharest; \\ ${ }^{2}$ Department of Medical Psychology, 'Carol Davila' University of Medicine and Pharmacy, \\ 050474 Bucharest; ${ }^{3}$ Department of Dermatology and Allergology, Elias Emergency University Hospital, \\ 011461 Bucharest; ${ }^{4}$ Dermatology Research Laboratory, 'Carol Davila' University of Medicine and Pharmacy, \\ 050474 Bucharest; ${ }^{5}$ Department of Dermatology, 'Prof. N. Paulescu' National Institute of Diabetes, \\ Nutrition and Metabolic Diseases, 011233 Bucharest; ${ }^{6}$ Department of Physiology, \\ 'Carol Davila' University of Medicine and Pharmacy, 050474 Bucharest, Romania
}

Received August 20, 2018; Accepted September 28, 2018

DOI: $10.3892 /$ etm.2018.7058

\begin{abstract}
There are complex interconnections between the nervous system and the skin highlighted by the impact of stress and neuroendocrine factors on various dermatological conditions. We investigated the influence of stress on skin neurogenic inflammation induced by capsaicin. A total of 31 healthy subjects were randomized into two groups: subjects in the stress group underwent a stress-inducing protocol and those in the control group were exposed to indifferent conditions. Subsequently, topical capsaicin cream was administered on the non-dominant anterior forearm of each subject from the two groups. The assessment of the local inflammatory reaction induced by capsaicin was performed by thermography at 25 and 40 min post-application. In both groups the inflammatory reaction induced by capsaicin was evidenced at $25 \mathrm{~min}$ and was maintained at $40 \mathrm{~min}$ post-application. However, at 40 min post-application the hyperthermal area was larger in subjects from the stress group, suggesting that stress exposure is associated with an amplification of the mechanisms involved in capsaicin-induced skin neurogenic inflammation.
\end{abstract}

\section{Introduction}

Stress has become a major component of modern lifestyle, the human body being frequently faced to the need for adaptation. Through its impact on the mechanisms of homeostasis, stress can alter health, stress-related illnesses being an alarming

Correspondence to: Dr Daniel Boda, Dermatology Research Laboratory, 'Carol Davila' University of Medicine and Pharmacy, 8 Eroii Sanitari Boulevard, 050474 Bucharest, Romania

E-mail: daniel.boda@yahoo.com

Key words: stress, capsaicin, neurogenic inflammation, thermography, skin conductance ongoing problem in developed countries, both through social costs and healthcare spending, but also economic losses from productivity decline (1-4).

Numerous studies have suggested that stress can be involved in triggering or aggravating various dermatological conditions, from skin cancer to inflammatory skin diseases such as psoriasis, atopic dermatitis, seborrheic eczema, acne, prurigo nodularis, lichen planus, chronic urticaria, rosacea and alopecia areata (5-15). The complexity of interconnections between the nervous system and the skin are surprising at the first glance; the action of stress on skin can be exerted both directly through the peripheral nervous system and indirectly through endocrine and immune systems.

Neurogenic inflammation is a particular type of inflammatory response, highlighting the multitude of neurocutaneous connections. The neurogenic inflammatory process developed in the skin is a potential factor involved in the onset and progression of some of the inflammatory dermatological diseases $(9,16)$. Hence, the study of mechanisms of activation and modulation of neurogenic inflammation has both clinical and fundamental importance.

Previous studies have highlighted the effect of stress on the mechanisms involved in the cutaneous neurogenic reaction, such as modulating the density and activity of nociceptive nerve fibers sensitive to capsaicin, modulating the activity of their neurons, increasing SP release from the unmyelinated nerve endings of the skin and the degranulation of cutaneous mast cells (17-21).

In this study, we aimed to assess the influence of stress on the capsaicin-induced skin inflammatory reaction, one of the most commonly used neurogenic skin inflammation models in scientific research (22-24). To achieve this goal we proposed a good control of unwanted external stressors. In order to induce psychological stress, we proposed the development and implementation of an easy-to-use protocol that contains various tasks capable of generating stress differently, thus allowing stress to be induced to as many categories of subjects as possible and avoiding the installation of habituation. We also sought to evaluate the capsaicin-induced inflammatory 
response by methods in which the subjective factor had the least possible involvement.

\section{Subjects and methods}

Subjects. In this study, 31 healthy subjects of both sexes $(\mathrm{M}=13, \mathrm{~F}=18)$ were enrolled, aged between 20 and 35 years (mean age 25.096 years).

The study was carried out with the approval of the Ethics Committee of Carol Davila University of Medicine and Pharmacy (Bucharest, Romania). At the time of the study, all participants attended university studies and enrollment in the study was done on a voluntary basis. The participation of the subjects in the study was conditioned by a written consent.

Subjects with cardiovascular or respiratory diseases, autoimmune diseases, neoplasms, organ transplants, allergic reactions to the substances used in the study, as well as subjects treated with drugs that may influence the physiological parameters studied were excluded from the study. Other exclusion criteria were major psychiatric diseases and major stress or infectious diseases in the previous month. Moreover, pregnant or lactating women were excluded from the study.

Study participants were asked to avoid drinking psychoactive substances, alcohol, coffee, tea, energy drinks or containing caffeine, smoking, medication, and intense physical effort $24 \mathrm{~h}$ before the test.

Subjects included in the study were randomized into two groups: stress group of 17 subjects $(F=10, M=7$, mean age $25.12 \pm 4.96$ years) undergoing a stress-inducing protocol; and control group of 14 subjects $(F=8, M=6$, mean age $25.07 \pm 4.32$ years) who were exposed to indifferent conditions.

Procedures. Experiments were performed during the afternoon (12:00 p.m. - 18:00 p.m.) and lasted approximately $2 \mathrm{~h}$ per subject. During the experiments, a temperature of $22 \pm 2^{\circ} \mathrm{C}$ and a humidity of $50 \pm 5 \%$ were maintained in the laboratory.

Upon arrival at the place of the experiment, the subjects were given a 15 -min period of accommodation with the laboratory conditions, completing the inclusion forms in the study.

In order to quantify the impact of stressors in everyday life prior to participating in the study, we assessed the level of perceived stress for each subject using the Perceived Stress Scale (PSS) questionnaire (25).

Subsequently, each of the subjects participated in a $30-$ min computerized stage in which the subjects in the stress group underwent a stress-inducing protocol, and those in the control group were exposed to indifferent conditions. The stress-inducing protocol contained three phases of similar duration: a phase of viewing images with negative emotional load; an arithmetic counter-clock test phase; and a counter-clock intelligence test phase. Exposure to indifferent conditions was accomplished by viewing images with no negative emotional load.

Both during the accommodation and during the experiments the subjects were placed in a sitting position on a comfortable chair.

For $1 \mathrm{~min}$ before and during the computerized stage, the skin conductance was recorded in each subject. At the end of this stage, each of the subjects quantified on a scale from 0 to 10 the level of stress experienced during exposure to the stress induction protocol and indifferent conditions, respectively.

At the end of the computerized stage, the subjects in both groups participated in the capsaicin exposure stage in which, at the middle third of the non-dominant anterior forearm, topical capsaicin cream was administered at $0.1 \%$. The cream was kept in contact with the skin of the subjects for $10 \mathrm{~min}$, after which it was removed. The assessment of the local inflammatory reaction induced by capsaicin was performed by cutaneous thermography, and the thermographic images taken at 25 and $40 \mathrm{~min}$ after the onset of capsaicin administration were analyzed.

Viewing images with/without negative emotional load. The phase of viewing images with negative emotional load in the stress-inducing protocol and exposure to indifferent conditions by viewing images without unpleasant connotations were performed using the IAPS image set (26). The images used in the experiment were chosen on the basis of the individual emotional, activation and dominance values indicated in the IAPS technical report and were divided into two sets: a set of images without negative emotional load that contained neutral, pleasant or intense pleasant images; and a set of negative emotional images that contained neutral, unpleasant or intense unpleasant images.

The images were displayed on the screen of a monitor using a software developed by our group (27). The image display interval was $7 \mathrm{sec}$ and was chosen experimentally so that the subjects were exposed for long enough to the visual stimulus in order to achieve a reaction and at the same time to avoid habituation.

Arithmetic counter-clock test. The arithmetic test phase of the stress induction protocol was based on a similar MIST stress test concept (28). It was accomplished through a program that displays on a monitor screen arithmetical operations that the subject is trained to solve mentally within a limited time-frame.

Counter-clock intelligence test. For the phase of the stress-inducing intelligence test, we used a computerized version of the Raven's Progressive Matrices test (29), which is a non-verbal evaluation of the level of general intelligence (30).

The Raven test was used in the experiment because the notion of psychological test and assessment of intelligence level, accompanied by the investigator's assertion that test results will also be important in the experiment, is an important stress factor. In addition, the time frame available to solve the problems was limited to $10 \mathrm{~min}$.

Evaluation of cutaneous conductance. The skin conductance was recorded via a Varioport-B portable acquisition system (Becker Meditec, Karlsruhe, Germany) in a 8-bit digital system (spectrum $=0-70 \mu \mathrm{sec}$ ) at a frequency of $250 \mathrm{~Hz}$. The recording was performed in a bipolar system using two circular-shaped $\mathrm{Ag} / \mathrm{AgCl}$ electrodes with a $10 \mathrm{~mm}$ diameter contact surface (Becker Meditec), with a constant $0.5 \mathrm{~V}$. The applied current had a low voltage so it could not be perceived by the subjects.

Electrode placement was performed with caution, and subjects were instructed to avoid changing position during the 
Table I. Comparative analysis of cutaneous conductance and self-assessed stress at the end of the computerized stage.

\begin{tabular}{lccccccccc}
\hline & \multicolumn{3}{c}{ Stress } & & \multicolumn{3}{c}{ Control } & \\
\cline { 2 - 3 } Parameters & Mean & SD & Range & & Mean & SD & Range & Level of significance \\
\hline Skin conductance (\% reference level) & 106.1919 & 4.7425 & 19.5609 & & 101.2734 & 3.8161 & 13.1926 & $0.0074^{\mathrm{a}}$ \\
Self-assessed stress & 4.7647 & 1.888 & 5.5 & & 0.9643 & 1.117 & 3 & $0.00001^{\mathrm{b}}$ \\
\hline
\end{tabular}

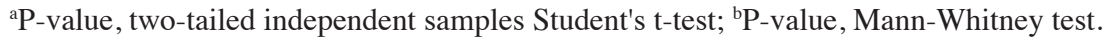

test to reduce the risk of electrode detachment due to movement and sweat secretion. The electrodes were positioned at the palm of the non-dominant hand, on the thenar and hypothenar eminences, ensuring a constant contact area and good contact between the electrodes and the skin surface (27). An isotonic paste with a composition close to that of the eccrine sweat secretion (31) was applied to the surface of the electrodes and they were applied to the skin using double-sided adhesive rings.

Prior to applying the electrodes, the skin was cleaned with water and soap, while avoiding skin abrasion maneuvers or contact with solvent agents. The level of skin conductance was determined for $1 \mathrm{~min}$ under baseline (reference) conditions. Then, recording of skin conductance continued throughout the computerized stage.

In 4 subjects, two in each lot, recording of skin conductance could not be completed due to technical reasons (recording artifacts or discontinuation of equipment activity).

For each subject, the average skin conductance level was calculated for the reference period and for the computerized stage. The average level of skin conductance for the computerized stage (stress induction protocol or exposure to indifferent conditions) was expressed as a percentage of basal level.

Administration of capsaicin. Capsaicin was topically applied as a $0.1 \%$ cream (CVS Pharmacy, Woonsocket, RI, USA). For each subject, $75 \mathrm{mg}$ of $0.1 \%$ capsaicin cream on a $3.142 \mathrm{~cm}^{2}$ cutaneous area was administered via a polycarbonate adhesive disc (Lucid Inc., Rochester, NY, USA). This capsaicin application method allowed isolation of the investigated region from the adjacent skin, an uniform administration of the same amount of active substance on a similar skin surface to all subjects and a better penetration of the active substance into the skin, generated by the occlusive effect induced by the plastic adhesive disc.

Evaluation of the inflammatory reaction by cutaneous thermography. Thermographic evaluation was performed with a FLIR ThermoVision A320 camera (Flir Systems, Danderyd, Sweden). The images were taken at a frequency of $30 \mathrm{~Hz}$ with a resolution of $320 \times 240$ pixels. The thermographic camera was fixed at a determined distance by the non-dominant forearm of each subject, and during the thermographic recording, subjects were asked to avoid altering the position of the forearm.

The thermographic images taken at 25 and 40 min after the onset of capsaicin application were analyzed. Inflammatory area measurement was performed using the Image $1.45 \mathrm{~s}$ image analysis program (http://rsbweb.nih.gov/ij/; National Institutes of Health, Bethesda, MD, USA). Two measurements were made for each subject and each experimental stage, while the value of the inflammatory area was expressed as the mean of the two measurements.

Statistical analysis. We used SPSS 15.0 software (SPSS, Inc., Chicago, IL, USA) and GraphPad Prism (Graphpad Software, Inc., San Diego, CA, USA) for statistical analysis of data. The confidence level between the two measurements of the capsaicin-induced inflammatory area, performed for each subject in the two groups and for each time interval, was estimated by the intraclass correlation coefficient (ICC) tests in a two-way mixed effects model using a definition of absolute agreement. To test the normality and homogeneity of the data distribution, Bartlett's test was used. The differences between the two groups were evaluated using two-tailed independent samples Student's t-test for normal and homogeneous distribution and Mann-Whitney U test when the data did not express a normal and homogeneous distribution. Within each of the two groups, an evaluation of the inflammatory area evolution was performed using the Wilcoxon signed-rank test. The results were presented as mean \pm standard deviation $(\mathrm{SD}) . \mathrm{P}<0.05$ values were considered to indicate statistically significant differences.

\section{Results}

Initially, a comparative analysis was performed in the two groups of the global level of perceived stress, using the PSS score. Control group subjects had a PSS score of 23.2857 \pm 5.2540 , and those in the stress group had a PSS score of $22.5882 \pm 4.9882$. There were no statistically significant differences between groups $(\mathrm{P}=0.7080)$.

Further, a comparative analysis of the self-assessed level of stress experienced during the computerized stage by the subjects of the two groups was carried out. Moreover, the effects of exposure to the stress induction protocol and indifferent conditions on skin conductance were also compared (Table I and Fig. 1).

The stress experienced during the computerized stage had a score of $0.9643 \pm 1.117$ in control group subjects and $4.7647 \pm 1.888$ in subjects in the stress group, the difference between the two groups being statistically significant $(\mathrm{P}=0.00001)$.

Regarding the skin conductance reference level, there were no statistically significant differences between the two groups $(\mathrm{P}=0.5125)$. In contrast, exposure to the stress induc- 
A

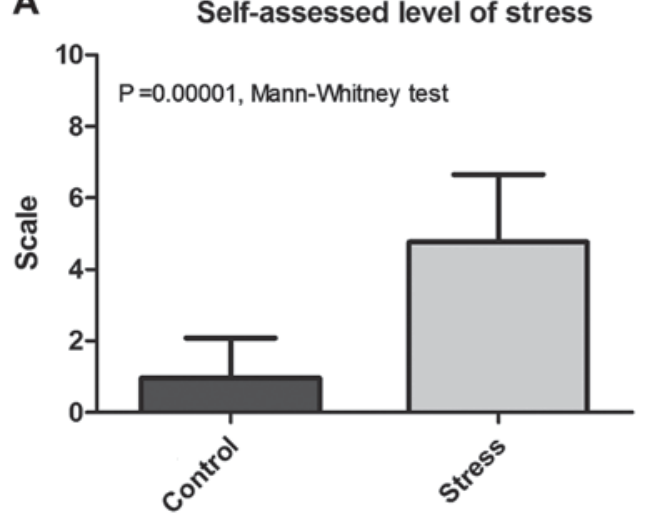

B

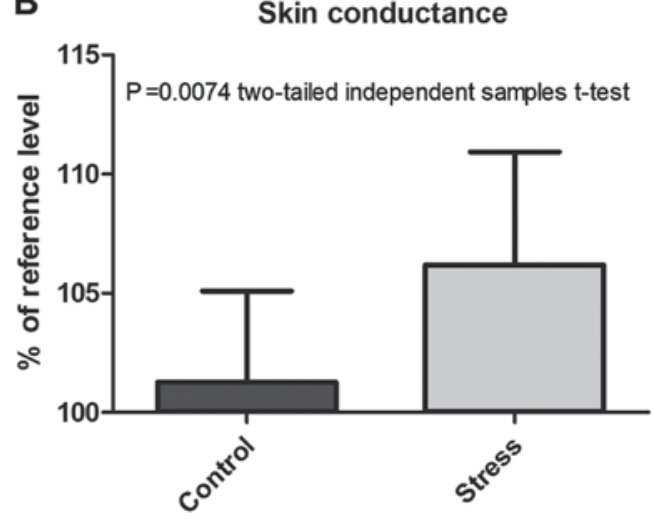

Figure 1. Comparative analysis of (A) self-assessed level of stress and (B) skin conductance during the computerized stage.

A

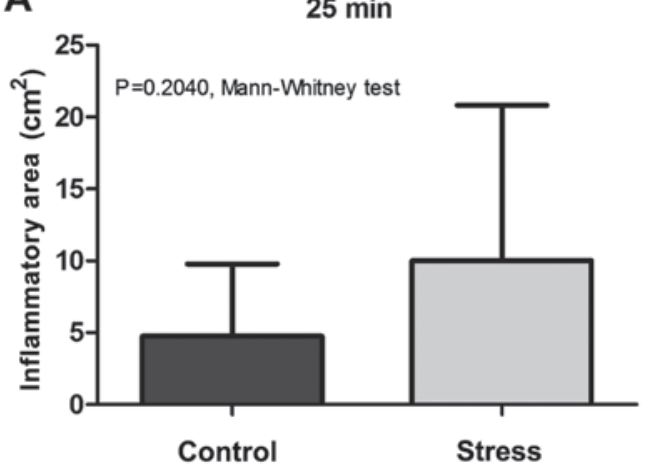

B

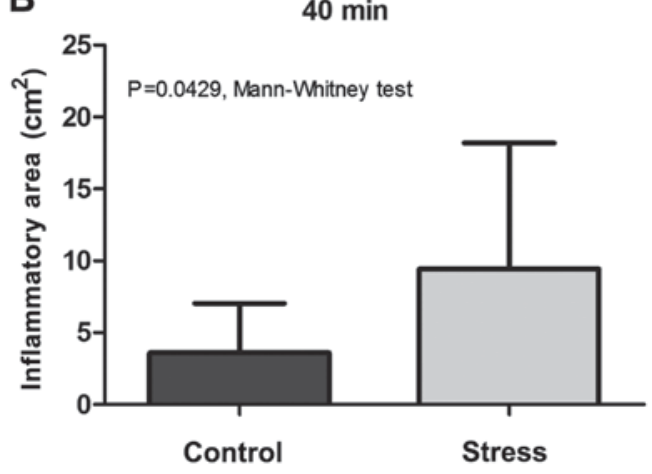

Figure 2. Comparative analysis of the inflammatory area evaluated at (A) 25 min and (B) 40 min after capsaicin administration.

tion protocol resulted in a statistically significant increase in the level of skin conductance. Thus, the average level of skin conductance during the computerized stage, expressed as a percentage of the reference level, was $106.1919 \pm 4.7425 \%$ for the stress group vs. $101.2734 \pm 3.8161 \%$ for control group $(\mathrm{P}=0.0074$ two-tailed independent samples t-test).

The evaluation of capsaicin-induced skin reaction was performed by analyzing in each group the evolution of the inflammatory area assessed by thermography at 25 and 40 min after capsaicin application, followed by quantification of the differences between the two groups for each of the above mentioned time intervals. The ICC of the capsaicin-induced inflammatory area measurements was 0.989 .

Within the control group, the inflammatory area evaluated $25 \mathrm{~min}$ after capsaicin administration was $4.7610 \pm 5.0133 \mathrm{~cm}^{2}$, while at $40 \mathrm{~min}$ post-application it appeared to slightly decrease to $3.5842 \pm 3.4560 \mathrm{~cm}^{2}$, without the difference between the two values reaching the level of statistical significance $(\mathrm{P}=0.064039$, two-tailed Wilcoxon signed ranks test).

In stress group, $25 \mathrm{~min}$ after application of capsaicin the inflammatory area had a value of $10.0063 \pm 10.8107 \mathrm{~cm}^{2}$ and at $40 \mathrm{~min}$ post-application was $9.4286 \pm 8.7749 \mathrm{~cm}^{2}$, the difference between the two values being not statistically significant $(\mathrm{P}=0.90579)$.

Comparative analysis between groups (Table II and Figs. 2 and 3) showed that even if at 25 min after capsaicin administration there were no statistically significant differences ( $\mathrm{P}=0.2040$, Mann-Whitney test), at $40 \mathrm{~min}$ post-treatment, the inflammatory area was significantly higher in the stress group, compared to control group $(\mathrm{P}=0.0429)$.

\section{Discussion}

Despite the great impact of neuroendocrine factors in dermatological conditions and the extensive research in animal or in vitro models, until now there are only a limited number of studies in human subjects regarding the role of stress in modulation of capsaicin-induced skin reactions. However, a recent study on healthy women showed that increased numbers of stressful life events are associated with a larger area of secondary hyperalgesia after topical application of capsaicin (32). Another investigation by Lutgendorf et al (33) on 50 healthy subjects of both sexes showed a decreased capsaicin-induced skin inflammation associated with stress reduction by relaxation techniques but could not reveal a direct impact of stress on this reaction. Another study of the same research group assessed the impact of stress and relaxation on the pain sensation induced by cutaneous administration of capsaicin, indicating that women undergoing relaxation experienced a significantly lower level of pain than those exposed to stress (34). Research has shown that stress reduction techniques can lessen the neurogenic inflammatory response induced by topical administration of capsaicin, the impact being different depending on the technique used $(35,36)$.

In our study regarding the stress influence on the capsaicininduced inflammatory neurogenic reaction we highlighted that 
Table II. Comparative analysis of the inflammatory area at 25 and 40 min after capsaicin application.

\begin{tabular}{|c|c|c|c|c|c|c|c|}
\hline \multirow[b]{2}{*}{ Parameters } & \multicolumn{3}{|c|}{ Stress } & \multicolumn{3}{|c|}{ Control } & \multirow[b]{2}{*}{ Level of significance ${ }^{a}$} \\
\hline & Mean & $\mathrm{SD}$ & Range & Mean & SD & Range & \\
\hline Inflammatory area at $25 \min \left(\mathrm{cm}^{2}\right)$ & 10.0063 & 10.8107 & 34.3709 & 4.7610 & 5.0133 & 17.8211 & 0.2040 \\
\hline Inflammatory area at $40 \mathrm{~min}\left(\mathrm{~cm}^{2}\right)$ & 9.4286 & 8.7749 & 28.9463 & 3.5842 & 3.4560 & 11.8757 & 0.0429 \\
\hline
\end{tabular}

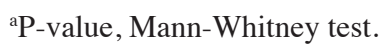
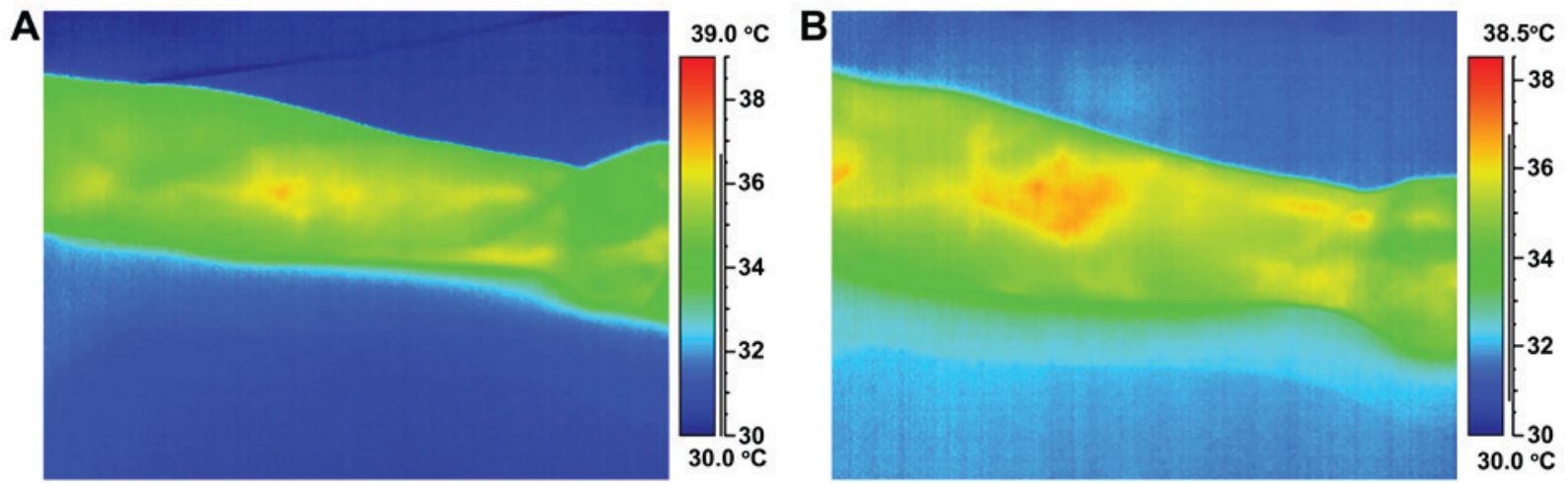

Figure 3. Thermographic recording of the inflammatory area $40 \mathrm{~min}$ after capsaicin administration to a subject from (A) Control group and (B) Stress group.

exposure to the stress induction protocol that we have implemented determined a significantly higher level of stress during the computerized stage. It also induced a significant increase of skin conductance, indicating an important sympathetic activation $(37,38)$. Within the subjects in both groups, topical application of capsaicin caused an inflammatory reaction that was evidenced by thermograpy at $25 \mathrm{~min}$ and was maintained at $40 \mathrm{~min}$ post-application. The hyperthermal area induced by topical application of capsaicin was higher at $40 \mathrm{~min}$ postapplication in subjects in the stress group, suggesting that acute stress exposure is associated with an extension of the area and duration of action of the mechanisms involved in capsaicin-induced skin neurogenic inflammation.

Previous studies have highlighted the important role played by dorsal root reflexes in the extension of the skin inflammation induced by capsaicin $(39,40)$. Stress may increase the capsaicin-induced cutaneous inflammatory response by facilitation of this type of reflexes by the modulator actions of catecholamines on the activity of nerve fibers sensitive to capsaicin and their neurons of origin $(17,18)$. The skin inflammatory response determined by capsaicin is dependent on the integrity of the sympathetic nervous system, its modulation being probably achieved through $\alpha 1$-adrenergic receptors $(41,42)$. Moreover, the increased inflammatory response induced by capsaicin correlates with the high serum level of norepinephrine (33).

Another way of acting through which stress could induce the amplification of the inflammatory area is to stimulate mast cell degranulation. The important role of mast cells in the capsaicin inflammatory response was highlighted in previous studies (43), and stress exposure determines a significant and rapid increase in the percentage of degranulated mast cells $(19,20)$.
In conclusion, our results show that acute stress exposure induces an amplification of the capsaicin-induced skin inflammatory response. It is likely that the influence of sympathetic activation and possibly other mechanisms, such as direct modulatory effects on mast cells, nociceptive nerve endings and their neurons of origin, would have a strong impact on the amplitude of capsaicin-induced inflammatory reaction in the skin.

\section{Acknowledgements}

Authors wish to thank Ioana Safta and Valentin Velican for their constant support during the course of this work.

\section{Funding}

This study was partially supported by a grant of Romanian Ministery of Research and Innovation, CCCDI-UEFISCDI (project no. 61PCCDI/2018 PN-III-P1-1.2-PCCDI-2017-0341) within PNCDI-III.

\section{Availability of data and materials}

The datasets used and/or analyzed during the current study are available from the corresponding author on reasonable request.

\section{Authors' contributions}

OG, AIM, IS, DB and CC were responsible for acquisition, analysis and interpretation of the data and contributed to drafting the manuscript and revising it critically for important 
intellectual content. All authors read and approved the final version of manuscript.

\section{Ethics approval and consent to participate}

This study was approved by the Ethics Committee of 'Carol Davila' University of Medicine and Pharmacy (Bucharest, Romania) and all participants provided written informed consent for the publication of data in this study.

\section{Patient consent for publication}

Not applicable.

\section{Competing interests}

The authors declare that they have no competing interests.

\section{References}

1. Lander F, Friche C, Tornemand H, Andersen JH and Kirkeskov L: Can we enhance the ability to return to work among workers with stress-related disorders? BMC Public Health 9: 372, 2009.

2. Dhabhar FS and McEwen BS: Enhancing versus suppressive effects of stress hormones on skin immune function. Proc Natl Acad Sci USA 96: 1059-1064, 1999.

3. Maddock C and Pariante CM: How does stress affect you? An overview of stress, immunity, depression and disease. Epidemiol Psichiatr Soc 10: 153-162, 2001.

4. Van Rhenen W, Blonk RW, van der Klink JJ, van Dijk FJ and Schaufeli WB: The effect of a cognitive and a physical stress-reducing programme on psychological complaints. Int Arch Occup Environ Health 78: 139-148, 2005.

5. Koo J and Lebwohl A: Psycho dermatology: The mind and skin connection. Am Fam Physician 64: 1873-1878, 2001.

6. Arck P and Paus R: From the brain-skin connection: The neuroendocrine-immune misalliance of stress and itch. Neuroimmunomodulation 13: 347-356, 2006.

7. Tausk F, Elenkov I and Moynihan J: Psychoneuroimmunology. Dermatol Ther 21: 22-31, 2008.

8. Gupta MA and Gupta AK: Psychiatric and psychological co-morbidity in patients with dermatologic disorders: Epidemiology and management. Am J Clin Dermatol 4: 833-842, 2003.

9. Căruntu C, Grigore C, Căruntu A, Diaconeasa A and Boda D: The role of stress in skin diseases. Medicina Internă: Internal Medicine 8: 73-84, 2011 (In Romanian).

10. Căruntu C, Ghiță MA, Căruntu A and Boda D: The role of stress in the multifactorial etiopathogenesis of acne. Ro Med J 58: 98-101, 2011

11. Surcel M, Constantin C, Caruntu C, Zurac S and Neagu M: Inflammatory cytokine pattern is sex-dependent in mouse cutaneous melanoma experimental model. J Immunol Res 2017: 9212134, 2017.

12. Caruntu C, Mirica A, Rosca AE, Mirica R, Caruntu A, Tampa M, Matei C, Constantin C, Neagu M, Badarau AI, et al: The role of estrogens and estrogen receptors in melanoma development and progression. Acta Endo 12: 234-241, 2016.

13. Caruntu C, Boda D, Constantin C, Caruntu A and Neagu M: Catecholamines increase in vitro proliferation of murine B16F10 melanoma cells. Acta Endocrinologica (Bucharest) 10: 545-558, 2014.

14. Lupu M, Caruntu A, Caruntu C, Papagheorghe LML, Ilie MA, Voiculescu V, Boda D, Constantin C, Tanase C, Sifaki M, et al: Neuroendocrine factors: The missing link in non-melanoma skin cancer (Review). Oncol Rep 38: 1327-1340, 2017.

15. Solomon I, Voiculescu VM, Caruntu C, Lupu M, Popa A, Ilie MA, Albulescu R, Caruntu A, Tanase C, Constantin C, et al: Neuroendocrine factors and head and neck squamous cell carcinoma: An affair to remember. Dis Markers 2018: 9787831, 2018.

16. Zegarska B, Lelińska A and Tyrakowski T: Clinical and experimental aspects of cutaneous neurogenic inflammation. Pharmacol Rep 58: 13-21, 2006.
17. Căruntu C, Boda D, Musat S, Căruntu A, Poenaru E, Calenic B, Savulescu-Fiedler I, Draghia A, Rotaru M and Badarau AI: Stress effects on cutaneous nociceptive nerve fibers and their neurons of origin in rats. Rom Biotechnol Lett 19: 9525-9538, 2014.

18. Filippi A, Caruntu C, Gheorghe RO, Deftu A, Amuzescu B and Ristoiu V: Catecholamines reduce transient receptor potential vanilloid type 1 desensitization in cultured dorsal root ganglia neurons. J Physiol Pharmacol 67: 843-850, 2016.

19. Singh LK, Pang $X$, Alexacos N, Letourneau $R$ and Theoharides TC: Acute immobilization stress triggers skin mast cell degranulation via corticotropin releasing hormone, neurotensin, and substance P: A link to neurogenic skin disorders. Brain Behav Immun 13: 225-239, 1999.

20. Căruntu C, Boda D, Musat S, Căruntu A and Mandache E: Stress-induced mast cell activation in glabrous and hairy skin. Mediators Inflamm 2014: 105950, 2014.

21. Pavlovic S, Daniltchenko M, Tobin DJ, Hagen E, Hunt SP, Klapp BF, Arck PC and Peters EM: Further exploring the brain-skin connection: Stress worsens dermatitis via substance P-dependent neurogenic inflammation in mice. J Invest Dermatol 128: 434-446, 2008.

22. Căruntu C, Negrei C, Ghiţă MA, Căruntu A, Bădărău AI, Buraga I, Boda D, Albu A and Branisteanu D: Capsaicin, a hot topic in skin pharmacology and physiology. Farmacia 63: 487-491, 2015.

23. Căruntu $\mathrm{C}$ and Boda D: Evaluation through in vivo reflectance confocal microscopy of the cutaneous neurogenic inflammatory reaction induced by capsaicin in human subjects. J Biomed Opt 17: 085003, 2012.

24. Ghiţă MA, Căruntu C, Rosca AE, Căruntu A, Moraru L, Constantin C, Neagu M and Boda D: Real-time investigation of skin blood flow changes induced by topical capsaicin. Acta Dermatovenerol Croat 25: 223-227, 2017.

25. Cohen S, Kamarck T and Mermelstein R: A global measure of perceived stress. J Health Soc Behav 24: 385-396, 1983.

26. Lang PJ, Bradley MM and Cuthbert BN: International affective picture system (IAPS): Affective ratings of pictures and instruction manual. Technical Report A-8. University of Florida, Gainesville, FL, 2008.

27. Safta I, Grigore O and Căruntu C: Emotion detection using psycho-physiological signal processing. 2011 7th International Symposium on Advanced Topics in Electrical Engineering (ATEE), May 12-14, 2011. https://ieeexplore.ieee.org/ document $/ 5952203$.

28. Dedovic K, Renwick R, Mahani NK, Engert V, Lupien SJ and Pruessner JC: The Montreal imaging stress task: Using functional imaging to investigate the effects of perceiving and processing psychosocial stress in the human brain. J Psychiatry Neurosci 30: 319-325, 2005.

29. Raven JC: Standardization of progressive matrices, 1938. Br J Med Psychol 19: 137-150, 1941.

30. Raven J: The Raven's progressive matrices: Change and stability over culture and time. Cognit Psychol 41: 1-48, 2000.

31. Schmidt S and Walach H: Electrodermal activity (EDA): State of the art measurement and techniques for parapsychological purposes. J Parapsychol 64: 139-163, 2000.

32. You DS, Creech SK and Meagher MW: Enhanced area of secondary hyperalgesia in women with multiple stressful life events: A pilot study. Pain Med 17: 1859-1864, 2016.

33. Lutgendorf S, Logan H, Kirchner HL, Rothrock N, Svengalis S, Iverson K and Lubaroff D: Effects of relaxation and stress on the capsaicin-induced local inflammatory response. Psychosom Med 62: 524-534, 2000.

34. Logan H, Lutgendorf S, Rainville P, Sheffield D, Iverson K and Lubaroff D: Effects of stress and relaxation on capsaicin-induced pain. J Pain 2: 160-170, 2001

35. Rosenkranz MA, Davidson RJ, Maccoon DG, Sheridan JF, Kalin NH and Lutz A: A comparison of mindfulness-based stress reduction and an active control in modulation of neurogenic inflammation. Brain Behav Immun 27: 174-184, 2013.

36. Rosenkranz MA, Lutz A, Perlman DM, Bachhuber DR Schuyler BS, MacCoon DG and Davidson RJ: Reduced stress and inflammatory responsiveness in experienced meditators compared to a matched healthy control group. Psychoneuroendocrinology 68: 117-125, 2016.

37. Storm H, Myre K, Rostrup M, Stokland O, Lien MD and Raeder JC: Skin conductance correlates with perioperative stress. Acta Anaesthesiol Scand 46: 887-895, 2002.

38. Lidberg L and Wallin BG: Sympathetic skin nerve discharges in relation to amplitude of skin resistance responses. Psychophysiology 18: 268-270, 1981. 
39. Lin Q, Li D, Xu X, Zou X and Fang L: Roles of TRPV1 and neuropeptidergic receptors in dorsal root reflex-mediated neurogenic inflammation induced by intradermal injection of capsaicin. Mol Pain 3: 30, 2007.

40. Lin Q, Wu J and Willis WD: Dorsal root reflexes and cutaneous neurogenic inflammation after intradermal injection of capsaicin in rats. J Neurophysiol 82: 2602-2611, 1999.

41. Lin Q, Zou X, Fang L and Willis WD: Sympathetic modulation of acute cutaneous flare induced by intradermal injection of capsaicin in anesthetized rats. J Neurophysiol 89: 853-861, 2003

42. Ren Y, Zou X, Fang L and Lin Q: Sympathetic modulation of activity in Adelta- and C-primary nociceptive afferents after intradermal injection of capsaicin in rats. J Neurophysiol 93 365-377, 2005.
43. Bíró T, Maurer M, Modarres S, Lewin NE, Brodie C, Acs G, Acs P, Paus R and Blumberg PM: Characterization of functional vanilloid receptors expressed by mast cells. Blood 91: 1332-1340, 1998.

(i) $\ominus$ This work is licensed under a Creative Commons Attribution-NonCommercial-NoDerivatives 4.0 International (CC BY-NC-ND 4.0) License. 